\title{
Evolução Clínica Tardia dos Stents Farmacológicos. Segurança e Eficácia até Cinco Anos do Registro DESIRE
}

\author{
Amanda Guerra de Moraes Rego Sousa ${ }^{1}$, José Ribamar Costa Junior ${ }^{1}$, Adriana Moreira ${ }^{1}$, \\ Ricardo Alves da Costa', Manuel Nicolas Cano', Galo Alfredo Maldonado Andrade', \\ Alexandre Antonio Cunha Abizaid', Fausto Feres' ${ }^{1}$, Luiz Alberto Piva e Mattos ${ }^{1}$, Rodolfo Staico', \\ Ricardo Pavanello', Enilton Sergio Tabosa do Egito', José Eduardo Moraes Rego Sousa'
}

\section{RESUMO}

Fundamentos: A expansão das indicações para o uso dos stents farmacológicos liberadores de sirolimus e de paclitaxel inclui lesões de complexidade crescente e populações com perfil clínico muito diferente daquele dos estudos randomizados fundamentais. O registro DESIRE foi idealizado para monitorar a efetividade e a segurança institucional dos stents farmacológicos disponíveis para uso clínico desde 2002, em nosso meio. Método e Resultados: Entre maio/2002 e março/2007, foram incluídos 2.043 pacientes (P) tratados com $\geq 1$ Cypher $^{\circledR}$ ou Taxus ${ }^{\circledR}$, no Hospital do Coração da Associação do Sanatório Sírio, em São Paulo - SP. A média das idades foi de $63,8 \pm 11,3$ anos; $76,6 \%$ eram do sexo masculino e $28,49 \%$ eram diabéticos. Um total de 2.415 lesões foram tratadas, sendo implantados 2.983 stents: 2.608 Cypher $^{\circledR}$ e 375 Taxus $^{\circledR}$. As tromboses agudas/subagudas ( $\leq 30$ dias); tardias (31 dias - 360 dias) e muito tardias (>360 dias) ocorreram em $0,34 \%, 0,73 \%$ e $0,34 \%$ dos pacientes, representando incidência global de $1,42 \%$. A taxa de eventos maiores, nesse período de evolução, foi baixa e igual a $8,6 \%$ (154 P), sendo 45 $(2,51 \%)$ óbitos cardíacos; $50(2,8 \%)$ infartos do miocárdio e $59(3,3 \%)$ novas revascularizações. Conclusões: $A$ análise até 5 anos de evolução dos pacientes do registro DESIRE demonstra efetividade elevada e alto grau de segurança dos stents farmacológicos, com taxas de eventos maiores, revascularização da lesão-alvo e de trombose, semelhantes às dos estudos randomizados.

DESCRITORES: Endoprótese, efeitos adversos. Angioplastia transluminal percutânea coronária. Trombose. Resultado de tratamento.
SUMMARY

\author{
Long-term Clinical Outcomes of \\ Drug Eluting Stents. Safety and Efficacy \\ up to Five Years of the DESIRE Registry
}

Background: Indications for the implantation of sirolimusand paclitaxel-eluting stents are expanding and include complex lesions and subsets of patients with clinical and demographic characteristics very different from those of early pivotal randomized trials. The DESIRE Registry was planned to monitor the safety of both Cypher $^{\circledR}$ and Taxus $^{\circledR}$ stent available in Brazil since 2002. Methods and Results: From May 2002 through March 2007, 2043 patients treated with one or more than one stent (either $\operatorname{Cypher}^{\circledR}$ or $\operatorname{Taxus}^{\circledR}$ ) at Hospital do Coração da Associação do Sanatório Sírio were included in this Registry. Mean age was 63.8 (11.3) years; $76.6 \%$ were male and $28.4 \%$ had diabetes. A total of 2,415 lesions were treated and 2,983 stents were implanted: 2,608 Cypher ${ }^{\circledR}$ and 375 Taxus $^{\circledast}$. Acute and subacute ( $\leq 30$ days); late thrombosis (31 days - 360 days) and very late thrombosis ( $>360$ days) occurred in $0.34 \%, 0.73 \%$ and $0.34 \%$ of the patients, adding up to a $1.42 \%$ overall rate. Likewise, the major adverse cardiac event rate was low and added up to $8.6 \%$ (154 patients), including: $45(2.51 \%)$ cardiac deaths; $50(2.8 \%)$ myocardial infarctions and $59(3.3 \%)$ of additional revascularizations. Conclusions: This long-term analysis of the DESIRE Registry shows the high efficacy and safety profiles of drug-eluting stents, with a low thrombosis rate, which is similar to those of randomized trials.

DESCRIPTORS: Stents, adverse effects. Angioplasty, transluminal, percutaneous coronary. Thrombosis. Treatment outcome.

\footnotetext{
1 Hospital do Coração - Associação do Sanatório Sírio, São Paulo, SP. Este trabalho recebeu o prêmio de melhor tema livre apresentado no XXIX Congresso da Sociedade Brasileira de Hemodinâmica e Cardiologia Intervencionista, Brasília, DF.

Correspondência: Amanda Guerra de Moraes Rego Sousa. Rua Desembargador Eliseu Guilherme, 123 - Paraíso - São Paulo - SP CEP 04004-030 - E-mail: amandars@uol.com.br Recebido em: 25/7/2007 • Aceito em: 14/8/2007
}

A pós os achados encorajadores do estudo $\mathrm{FIM}^{1,2}$ e de vários estudos randomizados fundamentais $^{2-9}$, demonstrou-se que os stents farmacológicos (SF) liberadores de sirolimus e de paclitaxel são altamente efetivos na redução das taxas de reestenose e de revascularização da lesão-alvo, quando comparados aos stents não recobertos. Apesar destes resultados terem sido obtidos em pacientes estáveis com lesões coronárias 
pouco complexas, selecionados a partir de critérios rigorosos de inclusão e exclusão, na rotina tem-se expandido amplamente as indicações para o seu uso, desde 2002, quando os SF tornaram-se disponíveis para uso clínico, no país.

Esta ampliação do uso da nova biotecnologia, para casos fora da bula, trouxe o questionamento sobre a efetividade e a segurança tardias dos SF, em pacientes de maior complexidade, e a dúvida se estes stents apenas retardariam ou efetivamente reduziriam o fenômeno da reestenose.

$\mathrm{Na}$ presente investigação, buscamos analisar os efeitos dos SF, numa grande coorte de casos, de um único centro hospitalar, com o intuito de avaliar os resultados dos SF num período de seguimento de até 5 anos de evolução clínica.

\section{MÉTODO}

\section{Casuística}

Entre maio de 2002 e março de 2007, foram incluídos, prospectiva e consecutivamente, todos os pacientes tratados com pelo menos um stent farmacológico, no Serviço de Cardiologia Intervencionista do Hospital do Coração da Associação do Sanatório Sírio, em São Paulo (SP). A esta coorte de casos deu-se o nome de Registro DESIRE (ㅁug Eluting Stents in the Real World).

Todos os pacientes com indicação de revascularização coronária percutânea foram incluídos, independentemente do quadro clínico ou angiográfico, desde que houvesse subvenção para o uso do stent farmacológico, pelos Seguros ou Planos de Saúde ou pelo próprio paciente. Foram excluídos apenas os casos com contra-indicações para o uso de qualquer um dos antiplaquetários previstos pelo protocolo farmacológico definido ou que necessitassem de cirurgia de outros aparelhos no curto prazo ( $\leq 6$ meses).

\section{Método}

O protocolo medicamentoso antitrombótico incluiu a combinação de dois agentes antiplaquetários com distintos sítios de ação: o ácido acetilsalicílico (AAS) e o clopidogrel. O pré-tratamento (AAS: 500 mg e clopidogrel: 300-600 mg) foi realizado 24 horas antes da intervenção, para os casos eletivos, ou de 3 a 6 horas antes do procedimento, para a síndrome coronária aguda sem supradesnivelamento do segmento ST. No caso de pacientes com infarto agudo do miocárdio nas primeiras horas de evolução, em que se pretendia realizar a recanalização mecânica primária da artéria culpada, administrou-se a dose de ataque dos dois medicamentos mencionados, aos quais se associou o uso do abciximab, que é um inibidor potente da glicoproteína IIb-IIla das plaquetas, de ação quase imediata.
Após o procedimento, o AAS foi mantido indefinidamente, na dose de 100-200 mg por dia e o clopidogrel, na dose de 75 mg diários, por 6 a 12 meses. Todos os pacientes foram insistentemente orientados para não descontinuarem a medicação antiplaquetária, a não ser sob orientação médica.

Foram utilizados, neste Registro, dois tipos de stents farmacológicos: stents Cypher ${ }^{\circledR}$, liberadores de sirolimus, e Stents Taxus $^{\circledR}$, liberadores de paclitaxel. A escolha do SF foi deixada a critério do investigador.

No stent Cypher ${ }^{\circledR}$, o sirolimus na formulação de liberação lenta, é adsorvido numa mistura de polímero não biodegradável de $5 \mathrm{~mm}$ de espessura, aplicada sobre a plataforma Bx Velocity ${ }^{\circledR}\left(\right.$ Cordis $\left.^{\circledR}, J \& J\right)$, que se constitui num stent tubular, multicelular, de aço inoxidável 316 L, perfurado a laser, sendo balão-expansível ${ }^{1,2}$. Mais recentemente, a modalidade utilizada foi o Cypher Select $^{\circledR}$, que representou uma evolução da plataforma original.

O stent Taxus $^{\circledR}$, com liberação lenta do fármaco paclitaxel, aprovado pelo FDA em 2004, demonstrou também ótimos resultados nos estudos fundamentais ${ }^{8,10}$. O paclitaxel, originalmente isolado da casca do taxus brevifolia, é um agente com potente atividade antitumoral, induzindo à redução de proliferação e da migração de células. Ele é altamente lipofílico, exercendo duradouro efeito celular, aumentando a estabilização dos microtúbulos não funcionantes ${ }^{11-14}$.

A técnica empregada para o implante dos SF foi a estabelecida desde os primórdios do uso desse novo instrumental, após a análise crítica dos resultados do estudo SIRIUS ${ }^{4}$. Sempre que julgada necessária, foi praticada a pré-dilatação cuidadosa da lesão-alvo, com 6 a 8 atm, visando a não traumatizar, com o balão, os segmentos adjacentes à lesão. A seguir, foi liberada a prótese previamente escolhida numa extensão tal que cobrisse toda a lesão-alvo, ultrapassando-a, proximal e distalmente, numa razão de extensões >1,2:1 (stent: lesão). Seguiu-se a pós-dilatação com altas pressões (14 a $16 \mathrm{~atm}$ ), que deveria ser praticada com balão mais curto que o stent, rigorosamente dentro da prótese, evitando-se qualquer barotrauma nas bordas não cobertas pela prótese. Se mais de um stent fosse necessário para cobrir toda a lesão, praticava-se a sobreposição de 2 a $4 \mathrm{~mm}$ das bordas distal da primeira prótese e proximal da segunda, evitando-se deixar qualquer ponto da lesão não coberto pelas hastes dos stents.

O procedimento foi monitorado pela angiografia coronária quantitativa, que definia, em qualquer etapa, o resultado obtido. A ultra-sonografia intracoronária foi utilizada quando a avaliação angiográfica não definiu como ótimo o implante do stent.

Considerou-se sucesso técnico, a obtenção de lesão residual $<20 \%$, sem que se verificassem trombos, alterações da velocidade do fluxo coronário ou dissecções nas bordas da prótese implantada. Caso algum 
desses eventos fosse observado, o controle desses achados era necessário, antes que se determinasse o término do procedimento.

Durante a hospitalização, todos os pacientes foram submetidos à avaliação eletrocardiográfica e dos marcadores bioquímicos (CK e CKMB), em três momentos: antes e imediatamente após a intervenção, assim como na alta hospitalar, que só foi confirmada na dependência da ausência de alterações significativas dessas avaliações.

Todos os pacientes foram acompanhados por meio de visitas clínicas; de contatos telefônicos (por pessoa habilitada e treinada para essa finalidade) e/ou de questionários. Os períodos preestabelecidos de avaliação foram: 1 mês, 6 meses e 12 meses de evolução, e anualmente, a partir de então, para informações sobre o quadro clínico e para a apuração de eventos cardíacos maiores (óbito, infarto do miocárdio e novas revascularizações), e também para monitoração do uso da terapêutica antiplaquetária protocolar.

Um banco de dados especialmente dedicado ao Registro DESIRE foi desenvolvido pelo analista de sistemas do nosso Serviço, onde constam dados pormenorizados das características clínicas, angiográficas e informações técnicas da intervenção de cada um dos pacientes registrados. Os dados evolutivos hospitalares e os obtidos nos períodos definidos $(1,6,12,24,36,48$ e 60 meses) após o procedimento-índice foram também registrados. As taxas de seguimento foram calculadas, considerando-se o número de pacientes acompanhados sobre o número de casos elegíveis que tivessem atingido aquela etapa pré-especificada do acompanhamento.

Os óbitos foram classificados como cardíacos e não cardíacos. Óbitos de causas indeterminadas foram relatados como cardíacos. Tendo como base as alterações do eletrocardiograma na evolução, a elevação da concentração enzimática (CKMB >2 vezes o valor normal) ou ambos os achados, foram classificados os infartos do miocárdio (IM) como: IM com onda Q, IM sem onda $\mathrm{Q}$ ou desconhecido, este quando nenhuma documentação fosse disponível para a determinação. Todas as reintervenções devido à recorrência da lesão dentro do stent implantado no procedimento-índice ou no segmento vascular, que incluía os $5 \mathrm{~mm}$ proximais e $5 \mathrm{~mm}$ distais ao stent, foram classificados como: revascularização da lesão-alvo (RLA). Outros procedimentos repetidos no mesmo vaso, mas à distância deste segmento, foram tidos como revascularização da lesão nãoalvo. A trombose do stent foi classificada de acordo com o proposto pelo Academic Research Consortium, como: definitiva (confirmação angiográfica ou anatomopatológica); provável (morte súbita $\leq 30$ dias pós-SF ou infarto relacionado à região da artéria tratada, mesmo sem confirmação angiográfica) e possível (morte súbita $>30$ dias pós-SF). Quanto à classificação temporal, as tromboses protéticas ainda foram divididas como: agudas e subagudas ( $\leq 30$ dias); tardias (31 dias - 360 dias) e muito tardias (>360 dias).
As variáveis contínuas foram apresentadas como média e desvio-padrão. As variáveis categóricas foram apresentadas em números e porcentuais. O teste $t$ de Student foi usado para comparação das médias e o teste $\chi^{2}$, para comparação entre as proporções. Valores da probabilidade $<0,05$ foram considerados indicativos de significância estatística.

\section{RESULTADOS}

Foram registrados, até março de 2007, 2.043 pacientes, que receberam $3.047 \mathrm{SF}$, sendo 2.608 (85\%) stents Cypher $^{\circledR}\left(\right.$ Cordis $^{\circledR}$, Johnson \& Johnson); 375 (12,3\%) stent Taxus ${ }^{\circledR}$ (Boston Scientific $^{\circledR}$ ) e $63(2,7 \%)$ outros SF, sendo a média de stents implantados por pacientes de 1,5. Para as finalidades dos desfechos, consideramos os dados referentes aos $1.792 \mathrm{P}$ tratados que alcançaram os seis meses de evolução pós-SF.

A média das idades desta amostra foi de 63,8 anos ( $\mathrm{DP}=11,3$ anos), sendo $76,6 \%$ dos pacientes do sexo masculino e $28,4 \%$ diabéticos $(6,3 \%$ dependentes do uso de insulina). Estas características de base estão pormenorizadas nas Tabelas 1 e 2 .

As extensões das próteses implantadas variaram de 8 a $33 \mathrm{~mm}$ e os diâmetros de 2,25 a 4,5 mm. A extensão mais freqüentemente usada foi $18 \mathrm{~mm}(41,9 \%)$ e o diâmetro mais freqüentemente escolhido, 3,0 mm $(35,9 \%)$. Os pormenores mais importantes relacionados aos vasos-alvo, às lesões tratadas e ao procedimento estão nas Tabelas 3 e 4.

No momento da alta, todos os pacientes, à exceção de um caso, estavam sendo tratados com a terapêutica antiplaquetária dupla (AAS + clopidogrel) nas doses prescritas. Este caso de suspensão mencionado apresentou hemorragia digestiva alta, durante a internação hospitalar, decorrente de úlcera gástrica, havendo indicação da retirada dos medicamentos protocolares. Evoluiu com IM, devido à trombose das próteses implantadas nas artérias descendente anterior e artéria coronária direita, vindo a falecer no hospital.

\section{TABELA 1}

\section{Características clínicas}

$\begin{array}{lr}\text { Idade (média, DP - anos) } & 63,8(11,3) \\ \text { Sexo feminino (n/\%) } & 419(23,4) \\ \text { História de: } & 379(21,1) \\ \text { Infarto prévio (n/\%) } & 450(25,1) \\ \text { Intervenção percutânea prévia (n/\%) } & 575(26,5) \\ \text { Cirurgia de revascularização prévia (n/\%) } & 509(28,4) \\ \text { Diabetes (n/\%) } & 129(6,3) \\ \text { Dependente de insulina (n/\%) } & 1349(77,2) \\ \text { Hipertensão (n/\%) } & 1086(60,6) \\ \text { Hipercolesterolemia (n/\%) } & 575(32,1) \\ \text { Tabagismo (n/\%) }\end{array}$


Os dados de seguimento foram obtidos de 1.738 (97\%) pacientes dos 1.792 tratados com mais de seis meses de evolução.

A trombose dos SF foi observada em 29 casos $(29 / 2.043=1,42 \%)$. Houve confirmação angiográfica em 14 (48\%) pacientes; um (4\%) caso foi considerado como provável trombose e os demais 14 (48\%) foram classificados como trombose possível. A distribuição temporal da trombose protética é a que se encontra na Figura 1. Dos casos com comprovação angiográfica, dois faleceram no hospital e 12 evoluíram com infarto do miocárdio. Dos 15 casos restantes, 11, segundo relato dos familiares, tiveram morte súbita e quatro, infarto do miocárdio, na evolução tardia.

Chama a atenção o fato de que 7 (24\%) desses eventos tenham ocorrido dentro do primeiro mês após o implante, 15 (52\%) entre 1 mês e 1 ano e 7 (24\%) após 360 dias. Vinte e quatro $(82,7 \%)$ casos de trombose não estavam observando a medicação antiplaquetária prescrita.

$\mathrm{Na}$ Tabela 5, estão descritos os eventos cardíacos maiores detectados no seguimento.

Nas Figuras 2 e 3, encontram-se as curvas de sobrevida livre de revascularização da lesão-alvo e livre de eventos cardíacos maiores.

\section{DISCUSSÃO}

Os resultados verificados no Registro DESIRE demonstram que o uso rotineiro dos SF Cypher ${ }^{\circledR}$ e Taxus $^{\circledR}$ é efetivo (baixas taxas de eventos cardíacos maiores e de revascularização da lesão-alvo) no curto e longo prazos,

TABELA 2

Características angiográficas

\begin{tabular}{lr}
\hline Número de vasos acometidos & \\
1 vaso (n/\%) & $779(43,5)$ \\
2 vasos (n/\%) & $576(32,1)$ \\
3 vasos/TCE (n/\%) & $437(24,4)$ \\
Indicações para o procedimento-índice & \\
Isquemia silenciosa (n/\%) & $528(29,5)$ \\
Angina estável (n/\%) & $538(30,0)$ \\
Angina instável (n/\%) & $456(25,4)$ \\
IAM em evolução (n/\%) & $270(15,0)$ \\
$\quad \leq 24$ h (n) & 55 \\
24 - 72 h (n) & 133 \\
$>72$ h (n) & $117(4,8)$ \\
Indicações especiais $(*)$ & $154(6,4)$ \\
Lesões reestenóticas (n/\%) & $131(5,4)$ \\
Bifurcações $(\mathrm{n} / \%)$ & $128(5,3)$ \\
Lesões ostiais $(\mathrm{n} / \%)$ & \\
Pontes de safena $(\mathrm{n} / \%)$ & \\
\hline
\end{tabular}

(*) Referências 6-9 dão substrato a estas indicações. e que os resultados dos estudos randomizados ${ }^{4,10}$ podem ser replicados na prática, a despeito da inclusão atual de pacientes com uma grande variedade de características clínicas e angiográficas de alto risco e complexidade.

O primeiro relato da efetividade e segurança tardias dos SF, em pacientes consecutivos não selecionados com doença complexa, veio do Registro RESEARCH de Rotterdam ${ }^{15}$, demonstrando que o uso dos stents Cypher ${ }^{\circledR}$ esteve associado a taxas significativamente mais baixas

TABELA 3

Características qualitativas dos vasos-alvo e das lesões tratadas

\begin{tabular}{lr}
\hline Vasos tratados (n) & 2166 \\
Número de vasos tratados por paciente & 1,21 \\
Tipo de vasos tratados (n/\%) & $924(42,7)$ \\
Descendente anterior & $611(28,2)$ \\
Circunflexa & $598(27,6)$ \\
Coronária direita & $32(1,5)$ \\
Tronco da coronária esquerda & 2415 \\
Lesões tratadas (n) & 1,3 \\
Número de lesões tratadas por paciente & 130 \\
Tipo de lesões tratadas* (n/\%) & 673 \\
A & 981 \\
B1 & 631 \\
B2 & \\
C & \\
\hline (*) Classificação da American Heart Association e do \\
American College of Cardiology (AHA/ACC). \\
\hline
\end{tabular}

Tabela 4

Aspectos quantitativos dos vasos e das lesões tratadas e dados dos procedimentos

Média dos diâmetros de referência

dos vasos tratados (DP/mm) 2,75 $(0,51)$

Média dos graus das estenoses (DP/\%)

Pré-procedimento

$64,8(16,3)$

Pós-procedimento

$5,55(4,17)$

Média das extensões da lesão-alvo (mm) 15,95 $(7,9)$

Número de stents implantados $\quad 2.983$

Número de stents por paciente $\quad 1,46$

Média das extensões dos stents

implantados (DP/mm) 19,03 $(5,73)$

Relação: extensão do stent/extensão da lesão $\quad 1,23$

Implante direto (n/\%) $1.350(55,9)$

Média da pressão máxima de

liberação (DP/atm)

$14,84(2,72)$

Pós-dilatação (n/\%)

$920(38,1)$

$\mathrm{DP}=$ desvio-padrão 
TABELA 5

Taxas de eventos cardíacos maiores na evolução até 5 anos, com média de seguimento clínico de $2,2 \pm 1,2$ anos

\begin{tabular}{lr}
\hline Óbito $(\mathrm{n} / \%)$ & $78(4,4)$ \\
Cardíaco (n/\%) & 45 \\
Não cardíaco (n/\%) & 33 \\
Infarto do miocárdio (n/\%) & $50(2,8)$ \\
Com onda Q (n/\%) & 11 \\
Sem onda Q (n/\%) & 39 \\
Revascularização da lesão-alvo (n/\%) & $59(3,3)$ \\
Intervenção percutânea (n/\%) & 43 \\
Cirurgia de revascularização miocárdica (n/\%) & 16 \\
Trombose aguda/subaguda, tardia e & \\
muito tardia (n/\%) & $29(1,42)$ \\
Aguda/Subaguda (n/\%) & $7(0,34)$ \\
Tardia (n/\%) & $15(0,73)$ \\
Muito Tardia (n/\%) & $7(0,34)$ \\
\hline
\end{tabular}

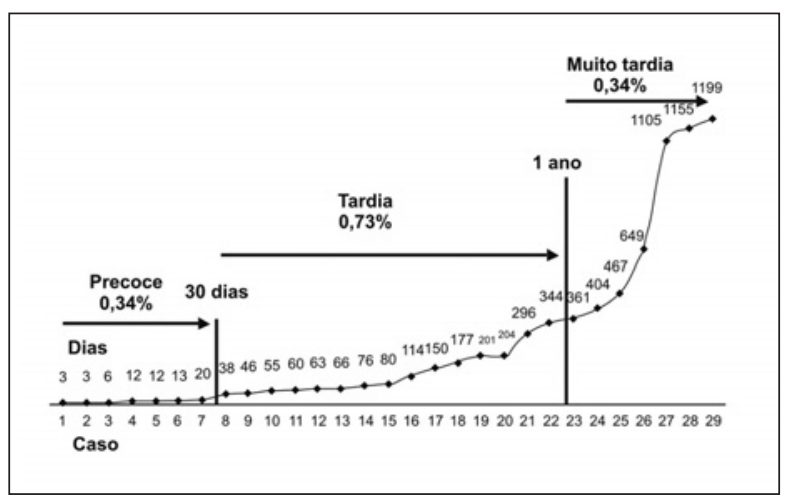

Figura 1 - Distribuição temporal da ocorrência do fenômeno de trombose protética em 29 pacientes $(1,42 \%)$ dos 2.043 incluídos no Registro DESIRE.

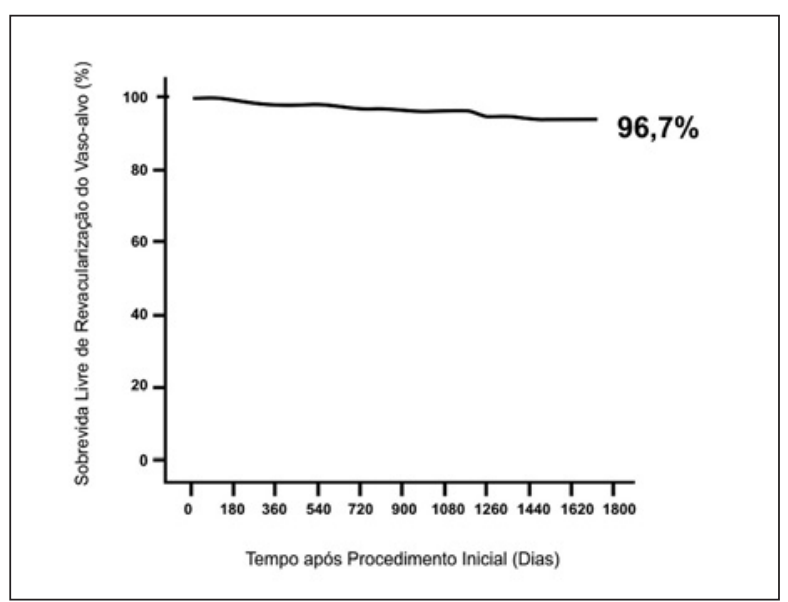

Figura 2 - Curva de sobrevida livre de revascularização do vasoalvo, até cinco anos de evolução dos pacientes do Registro DESIRE, incluídos de maio de 2002 a março de 2007.

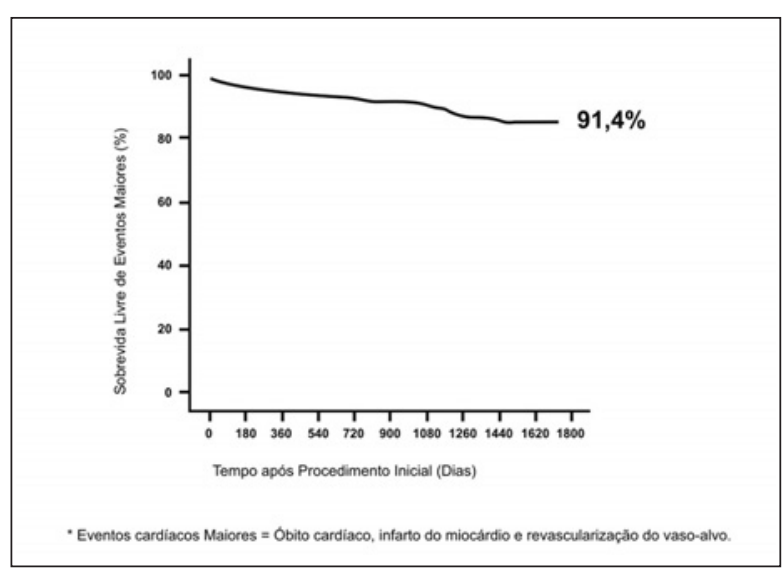

Figura 3 - Curva de sobrevida livre de eventos cardíacos maiores (óbito cardíaco, infarto agudo do miocárdio e revascularização do vaso-alvo) até cinco anos de evolução, dos pacientes do Registro DESIRE, incluídos de maio de 2002 a março de 2007.

de eventos cardíacos maiores e de revascularização do vaso-alvo do que o uso dos stents não recobertos, em pacientes ainda com evolução não longa. Vale ressaltar, entretanto - fato que os próprios autores enfatizam, numa avaliação de até dois anos ${ }^{16}$ - que tendo sido incluídos apenas 508 casos naquela coorte, a eventual observação de complicações tardias raras e não esperadas exigiam amostras mais amplas de casos e acompanhamentos mais tardios. O Registro DESIRE insere-se, portanto, no contexto desta exigência, oferecendo importante informação complementar aos estudos randomizados e aos pequenos registros, numa época em que a penetração dos SF cresce em todo o mundo, inclusive na América Latina e no Brasil (*).

A preocupação de que os SF apenas retardariam o fenômeno da reestenose, como se observou com a braquiterapia ${ }^{17}$, tem sido progressivamente descartada pela evidência da sustentabilidade dos resultados precoces obtidos, nos seguimentos clínicos mais longos ${ }^{1,2,7}$, como o da presente investigação, que chega, nos casos iniciais, a cinco anos de acompanhamento.

Até onde se sabe, comparados aos stents não recobertos, os SF não mudam a sobrevida, mas melhoram a qualidade de vida em decorrência da redução da reestenose e da necessidade de novas internações, investigações diagnósticas e revascularizações, o que deve contrabalançar os eventuais e raros efeitos negativos do perfil de segurança.

As taxas de trombose protética observadas no Registro DESIRE $(1,42 \%)$ são semelhantes àquelas observadas nas séries históricas com os stents metálicos não recobertos, dentro do primeiro ano de evolução ${ }^{18-21}$.

\footnotetext{
* Sousa A. DES penetration in Latin America. Apresentado ao XIII Congresso SOLACI '07. Buenos Aires, Argentina.
} 
Verifica-se, no entanto, com os SF, um adicional fenômeno trombótico muito tardio, da ordem de $0,34 \%$, a partir do primeiro ano de seguimento, sendo que em cerca da metade destes casos, a ocorrência foi entre o primeiro e segundo ano evolutivo e a outra metade entre o segundo e o terceiro ano. Outros estudos envolvendo

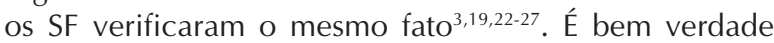
que os casos definidos como trombose muito tardia, no DESIRE, não tiveram, qualquer um deles, evidências de certeza, quer angiográfica ou anátomo-patológica, sendo os sete casos classificados como "possíveis tromboses", pois não se pôde destacar por completo a possibilidade do fenômeno. Por outro lado, a observação de que os SF retardam um pouco mais a cicatrização da lesão tratada em relação aos stents não recobertos ${ }^{28}$, associada aos achados de um resíduo de possível trombose muito tardia ( $>1$ ano), enfatizam a necessidade de manutenção da terapêutica antiplaquetária dupla (AAS + clopidogrel), por pelo menos um ano de duração, conduta recomendada mais recentemente, após o painel do FDA, nos Estados Unidos, ocasião em que os dados do Registro DESIRE foram apresentados.

Em contraste com os estudos prévios, com observação clínica mais curta $^{19,22}$, a presente série e alguns outros estudos ${ }^{16,29}$ têm se preocupado em ampliar o acompanhamento sistemático para pelo menos 12 ou até 24 meses pós-SF. Este período mais longo de observação afasta possíveis preocupações relacionadas:

- à endotelização retardada ${ }^{30,31}$;

- à excessiva trombose muito tardia ${ }^{32}$;

- a complicações relacionadas à liberação do medicamento ${ }^{32,33}$;

- a complicações devidas a outros moduladores da proliferação fibrointimal, tal como aconteceu com a braquiterapia ${ }^{34}$;

- a eventuais fenômenos de reestenose, incidente em períodos evolutivos mais avançados ${ }^{17}$.

Enfatiza-se, por fim, que os elevados porcentuais de obtenção de informações evolutivas (>96\%) do Registro DESIRE, com a captação de informações e monitoração periódica dos casos, feitas por profissionais habilitados e treinados para esta finalidade, reveste os achados da validade e da confiabilidade de que o emprego, a larga-mão, da nova biotecnologia requer.

Compararem-se registros com dados de estudos randomizados, como se sabe, é intrinsecamente impróprio. Contudo, os registros têm a virtude de, por serem menos restritivos nas inclusões e conter dados de uma grande gama e coorte de casos, refletirem os desafios que a versatilidade dos pacientes da prática clínica, que se enfrenta na rotina, oferecem.

\section{REFERÊNCIAS BIBLIOGRÁFICAS}

1. Sousa JE, Costa MA, Abizaid A, Feres F, Seixas AC, Tanajura $L F$, et al. Four-year angiographic and intravascular ultra- sound follow-up of patients treated with sirolimus-eluting stents. Circulation. 2005;111(18):2326-9.

2. Sousa JE, Costa MA, Farb A, Abizaid A, Sousa A, Seixas $A C$, et al. Vascular healing 4 years after the implantation of sirolimus-eluting stent in humans: a histopathological examination. Circulation. 2004;110:e5-6.

3. Morice MC, Serruys PW, Sousa JE, Fajadet J, Ban Hayashi E, Perin M, RAVEL Study Group. Randomized study with the sirolimus-coated Bx Velocity balloon-expandable stent in the treatment of patients with de novo native coronary artery lesions. A randomized comparison of a sirolimuseluting stent with a standard stent for coronary revascularization. N Engl J Med. 2002;346(23):1773-80.

4. Moses JW, Leon MB, Popma JJ, Fitzgerald PJ, Holmes DR, O'Shaughnessy C, SIRIUS Investigators. Sirolimus-eluting stents versus standard stents in patients with stenosis in a native coronary artery. N Engl J Med. 2003;349(14):1315-23.

5. Schofer J, Schluter M, Gershlick AH, Wijns W, Garcia E, Schampaert E, E-SIRIUS Investigators. Sirolimus-eluting stents for treatment of patients with long atherosclerotic lesions in small coronary arteries: double-blind, randomised controlled trial (E-SIRIUS). Lancet. 2003;362(9390):1093-9.

6. Schampaert E, Cohen EA, Schluter M, Reeves F, Traboulsi M, Title LM, C-SIRIUS Investigators. The Canadian study of the sirolimus-eluting stent in the treatment of patients with long de novo lesions in small native coronary arteries (CSIRIUS). J Am Coll Cardiol. 2004;43(6):1110-5.

7. Fajadet J, Morice MC, Bode C, Barragan P, Serruys PW, Wijns W, et al. Maintenance of long-term clinical benefit with sirolimus-eluting coronary stents: three-year results of the RAVEL trial. Circulation. 2005;111(8):1040-4.

8. Stone GW, Ellis SG, Cox DA, Hermiller J, O'Shaughnessy C, Mann JT, TAXUS IV Investigators. A polymer-based, paclitaxel-eluting stent in patients with coronary artery disease. $N$ Engl J Med. 2004;350(3):221-31.

9. Stone GW, Ellis SG, O'Shaughnessy CD, Martin SL, Satler L, McGarry T, TAXUS V ISR Investigators. Paclitaxel-eluting stents versus vascular brachytherapy for in-stent restenosis within bare-metal stents: the TAXUS V ISR randomized trial. JAMA. 2006;295(11):1253-63.

10. Ellis SG, Popma JJ, Lasala JM, Koglin JJ, Cox DA, Hermiller J, et al. Relationship between angiographic late loss and target lesion revascularization after coronary stent implantation: analysis from the TAXUS IV trial. J Am Coll Cardiol. 2005;45(8):1193-200.

11. Rowinsky EK, Donehower RC. Paclitaxel (taxol). N Engl J Med. 1995;332(15):1004-14.

12. Axel DI, Kunert W, Goggelmann C, Oberhoff M, Herdeg C, Kuttner A, et al. Paclitaxel inhibits arterial smooth muscle cell proliferation and migration in vitro and in vivo using local drug delivery. Circulation. 1997;96(2):636-45.

13. Baumbach A, Herdeg $C$, Kluge $M$, Oberhoff $M$, Lerch $M$, Haase KK, et al. Local drug delivery: impact of pressure, substance characteristics, and stenting on drug transfer into the arterial wall. Catheter Cardiovasc Interv. 1999;47(1):102-6.

14. Farb A, Heller PF, Shroff S, Cheng L, Kolodgie FD, Carter AJ, et al. Pathological analysis of local delivery of paclitaxel via a polymer-coated stent. Circulation. 2001;104(4):473-9.

15. Lemos PA, Hoye A, Goedhart D, Arampatzis CA, Saia F, van der Giessen WJ, et al. Clinical, angiographic, and procedural predictors of angiographic restenosis after sirolimus-eluting stent implantation in complex patients: an evaluation from the Rapamycin-Eluting Stent Evaluated At Rotterdam Cardiology Hospital (RESEARCH study). Circulation. 2004;109(11): 1366-70

16. Ong AT, van Domburg RT, Aoki J, Sonnenschein K, Lemos PA, Serruys PW. Sirolimus-eluting stents remain superior to 
bare-metal stents at two years: medium-term results from the Rapamycin-Eluting Stent Evaluated at Rotterdam Cardiology Hospital (RESEARCH) Registry. J Am Coll Cardiol. 2006;47(7): 1356-60.

17. Feres F, Muñoz J, Abizaid A, Staico R, Kuwabara M, Mattos $\mathrm{L}$, et al. Angiographic and intravascular ultrasound findings of the late catch-up phenomenon after intracoronary betaradiation for the treatment of in-stent restenosis. J Invasive Cardiol. 2005;17(9):473-7.

18. Cutlip DE, Baim DS, Ho KK, Popma JJ, Lansky AJ, Cohen DJ, et al. Stent thrombosis in the modern era: a pooled analysis of multicenter coronary stent clinical trials. Circulation. 2001;103(15):1967-71.

19. Ong AT, Hoye A, Aoki J, van Mieghem CA, Rodriguez Granillo GA, Sonnenschein K, et al. Thirty-day incidence and six-month clinical outcome of thrombotic stent occlusion after bare-metal, sirolimus, or paclitaxel stent implantation. J Am Coll Cardiol. 2005;45(6):947-53.

20. Bertrand ME, Legrand V, Boland J, Fleck E, Bonnier J, Emmanuelson $\mathrm{H}$, et al. Randomized multicenter comparison of conventional anticoagulation versus antiplatelet therapy in unplanned and elective coronary stenting: the full anticoagulation versus aspirin and ticlopidine (FANTASTIC) study. Circulation. 1998;98(16):1597-603.

21. Serruys PW, Unger F, Sousa JE, Jatene A, Bonnier HJ, Schonberger JP, et al. Arterial Revascularization Therapies Study Group. Comparison of coronary-artery bypass surgery and stenting for the treatment of multivessel disease. $\mathrm{N}$ Engl J Med. 2001;344(15):1117-24.

22. Jeremias A, Sylvia B, Bridges J, Kirtane AJ, Bigelow B, Pinto DS, et al. Stent thrombosis after successful sirolimus-eluting stent implantation. Circulation. 2004;109(16):1930-2.

23. Colombo A, Moses JW, Morice MC, Ludwig J, Holmes DR Jr, Spanos V, et al. Randomized study to evaluate sirolimuseluting stents implanted at coronary bifurcation lesions. Circulation. 2004;109(10):1244-9.

24. Moreno R, Fernandez C, Hernandez R, Alfonso F, Angiolillo DJ, Sabaté $M$, et al. Drug-eluting stent thrombosis: results from a pooled analysis including 10 randomized studies. J Am Coll Cardiol. 2005;45(6):954-9.
25. Babapulle MN, Joseph L, Bélisle P, Brophy JM, Eisenberg MJ. A hierarchical Bayesian meta-analysis of randomised clinical trials of drug-eluting stents. Lancet. 2004;364(9434):583-91.

26. Chieffo A, Bonizzoni E, Orlic D, Stankovic G, Rogacka R, Airoldi $F$, et al. Intraprocedural stent thrombosis during implantation of sirolimus-eluting stents. Circulation. 2004;109 (22):2732-6.

27. Goy JJ, Urban P, Seydoux C, De Benedetti E, Stauffer JC. Use of sirolimus-eluting coronary stents in routine clinical practice. Catheter Cardiovasc Interv. 2004;62(1):26-9.

28. Joner M, Finn AV, Farb A, Mont EK, Kolodgie FD, Ladich $\mathrm{E}$, et al. Pathology of drug-eluting stents in humans: delayed healing and late thrombosis risk. J Am Coll Cardiol. 2006;48(1):193-202.

29. Stone GW, Ellis SG, Cox DA, Hermiller J, O'Shaughnessy C, Mann JT, TAXUS-IV Investigators. One-year clinical results with the slow-release, polymer-based, paclitaxel-eluting TAXUS ${ }^{\circledR}$ stent: the TAXUS-IV trial. Circulation. 2004;109(16):1942-7.

30. Suzuki T, Kopia G, Hayashi S, Bailey LR, Llanos G, Wilensky $R$, et al. Stent-based delivery of sirolimus reduces neointimal formation in a porcine coronary model. Circulation. 2001;104 (10):1188-93.

31. Guagliumi G, Farb A, Musumeci G, Valsecchi O, Tespili M, Motta $\mathrm{T}$, et al. Images in cardiovascular medicine: sirolimuseluting stent implanted in human coronary artery for 16 months: pathological findings. Circulation. 2003;107(9):1340-1.

32. Virmani R, Guagliumi G, Farb A, Musumeci G, Grieco N, Motta $\mathrm{T}$, et al. Localized hypersensitivity and late coronary thrombosis secondary to a sirolimus-eluting stent: should we be cautious? Circulation. 2004;109(6):701-5.

33. McFadden EP, Stabile E, Regar E, Cheneau E, Ong AT, Kinnaird $\mathrm{T}$, et al. Late thrombosis in drug-eluting coronary stents after discontinuation of antiplatelet therapy. Lancet. 2004;364(9444):1519-21.

34. Waksman R, Ajani AE, Pinnow E, Cheneau E, Leborgne L, Dieble $R$, et al. Twelve versus six months of clopidogrel to reduce major cardiac events in patients undergoing gammaradiation therapy for in-stent restenosis: Washington Radiation for In-Stent restenosis Trial (WRIST) 12 versus WRIST PLUS. Circulation. 2002;106(7):776-8. 